\title{
Avaliação da bomba muscular da panturrilha em pacientes portadores de varizes primárias dos membros inferiores através da pletismografia a ar
}

\author{
Assessment of calf muscle pump in patients with primary varicose \\ veins of the lower limbs by air plethysmography
}

\author{
André de Araújo Sacchi' ${ }^{1}$ Aldemar Araújo Castro², Guilherme Benjamin Brandão Pitta ${ }^{3}$, \\ Fausto Miranda Junior ${ }^{4}$
}

\section{Resumo}

Objetivo: Avaliar a influência do refluxo das veias musculares da panturrilha (surais e geniculares) na função da bomba muscular da panturrilha em pacientes com varizes primárias nos membros inferiores.

Métodos: Estudo transversal prospectivo, no qual os pacientes foram avaliados por meio de exame físico, mapeamento dúplex e pletismografia a ar. Foram selecionados 120 pacientes divididos em dois grupos (60 indivíduos em cada). O primeiro grupo apresentava refluxo das veias musculares da panturrilha e o segundo grupo apresentava ausência de refluxo. Cada grupo foi analisado com pletismografia a ar para estudo da função da bomba da panturrilha. As variáveis estudadas foram fração de ejeção, fração do volume residual, volume ejetado e índice de enchimento venoso para membro inferior direito e esquerdo.

Resultados: No grupo com refluxo, índices de fração de ejeção abaixo de $60 \%$ (com p < 0,001) foram encontrados em $82,3 \%$ (em perna esquerda) e em 74,6\% (em perna direita) dos casos. Índices de fração do volume residual acima de $60 \%$ foram identificados em $62,5 \%$ (em perna esquerda, $p=0,015)$ e em $86,7 \%$ (em perna direita, $p=0,014)$ dos casos avaliados. Não houve variação estatisticamente significativa para o índice de enchimento venoso entre os grupos com e sem refluxo, com $p=0,140$ e $p=0,140$ para pernas esquerda e direita, respectivamente. Foram encontrados $63,6 \%$ dos pacientes com refluxo das veias da panturrilha (em perna esquerda), e $61,8 \%$ (em perna direita) com volume ejetado acima de $150 \mathrm{~mL}(\mathrm{p}=0,001)$.

Conclusões: Houve alteração da bomba muscular da panturrilha, levando a uma diminuição de sua função, em ambos os membros inferiores por conseqüência da presença do refluxo das veias musculares da panturrilha (geniculares e surais).

Palavras-chave: Pletismografia a ar, bomba muscular da panturrilha, varizes, veias gastrocnêmias e soleares, mapeamento dúplex.

\begin{abstract}
Objective: This article aims at assessing the influence of calf muscle vein reflux (sural and genicular veins) on calf muscle pump function in patients with primary varicose veins of the lower limbs.

Methods: Cross-sectional and prospective study assessing 120 patients divided into two groups (60 subjects each) by physical examination, duplex scan and air plethysmography. The first group showed calf muscle venous reflux, whereas the second group presented absence of reflux. Both groups were examined by air plethysmography to verify calf pump function through measurement of ejection fraction, residual volume fraction, ejected volume and venous filling index in both limbs.
\end{abstract}

Results: In the group of patients with reflux, ejection fraction indexes lower than $60 \%(\mathrm{p}<0.001)$ were found in $82.3 \%$ (left leg) and $74.6 \%$ (right leg) of cases. Levels of residual volume fraction greater than $60 \%$ were identified in $62.5 \%$ (left leg, $p=0.015$ ) and in $86.7 \%$ (right leg, $\mathrm{p}=0.014$ ) of assessed cases. There was no statistically significant variation concerning the venous filling index between groups with or without reflux, with $\mathrm{p}=0.140$ in both legs. Of all patients, $63.6 \%$ had calf vein reflux (left leg) and 61.8\% (right leg) had ejection volume greater than $150 \mathrm{~mL}(\mathrm{p}=0.001)$.

Conclusions: The calf pump function decreased in both lower limbs, due to presence of calf muscle vein reflux (genicular and sural veins).

Keywords: Air plethysmography, calf pump function, varicose veins, gastrocnemius and soleal veins, duplex scan.

1. Mestre. Professor assistente, Departamento de Cirurgia, Universidade Federal de Campina Grande (UFCG), Campina Grande, PB.

2. Mestre. Professor assistente, Departamento de Medicina Social, Universidade Estadual de Ciências da Saúde de Alagoas/Escola de Ciências Médicas de Alagoas (UNCISAL/ECMAL), Maceió, AL.

3. Doutor. Professor adjunto, Departamento de Cirurgia, UNCISAL/ECMAL, Maceió, AL.

4. Professor Titular de Cirurgia Vascular da Universidade Federal de São Paulo. (UNIFESP), São Paulo, SP.

Artigo submetido em 06.11.06, aceito em 30.01.07.

J Vasc Bras 2007;6(1):25-34.

Copyright $\odot 2007$ by Sociedade Brasileira de Angiologia e de Cirurgia Vascular 


\section{Introdução}

As veias varicosas são a manifestação mais comum da insuficiência venosa crônica ${ }^{1}$. Acredita-se que elas originam-se da distensibilidade anormal da parede venosa. Trabalhos mais antigos já sugeriam que as veias em pacientes com varicosidades eram mais distensíveis do que as veias em pacientes normais ${ }^{2}$, indicando provável base sistêmica para essa anormalidade. As varizes geralmente iniciam-se no ponto onde as veias superficiais comunicam-se com as profundas, particularmente nas junções safeno-femoral e safeno-poplítea e nos sistemas de veias perfurantes, devido à insuficiência valvular. As varizes primárias resultam da dilatação venosa sem trombose prévia, enquanto as secundárias são causadas por dano valvular decorrente de trombose venosa profunda em que a recanalização da obstrução leva à incompetência das veias profundas e perfurantes. Uma deficiente drenagem venosa, além do aumento da pressão venosa, elevará a pressão transmural em capilares e vênulas, produzindo alterações cutâneas, exsudação de líquidos, edema e deficiência na nutrição tecidual, o que favorece processos inflamatórios, infecciosos, tromboses e necrose tecidual levando à lipodermatoesclerose e a eventuais ulcerações ${ }^{3}$. As causas de refluxo no sistema venoso profundo podem ser de origem pós-trombótica, mas também podem ser idiopáticas. A flebografia descendente mostrou, em casos de origem idiopática, que o refluxo foi decorrente de delgadas cúspides valvares, agenesia ou aplasia val$\operatorname{var}^{4,5}$.

Através de uma detalhada história clínica, podemos identificar sinais e sintomas que nos fornecerão informações importantes quanto à origem, extensão e severidade do problema venoso, bem como identificar outras possíveis doenças relacionadas.

Podem ser requeridos exames complementares nãoinvasivos (e até mesmo invasivos) com o propósito de confirmar a avaliação clínica. O clássico teste do garrote pode nos revelar informações sobre prováveis pontos de refluxo do sistema profundo para o superficial, mas apresenta dificuldades na interpretação quando há veias varicosas não proeminentes. $O$ teste também não identifica se há obstrução ou refluxo no sistema venoso profundo.

Uma vez que a história e o exame clínico nem sempre indicam a natureza e a extensão das anormalidades referidas pelos pacientes, exames não-invasivos têm sido desenvolvidos para melhor investigar esses fatores ${ }^{6,7}$. Esses exames têm como características as avaliações qualitativa e quantitativa da hemodinâmica venosa e oferecem respostas à maioria das questões encontradas na prática clínica diária ${ }^{8}$. Eles podem ser empregados, por exemplo, para elucidar se há disfunção na bomba muscular da panturrilha e para determinar a extensão e severidade da obstrução e do refluxo venoso.

O objetivo deste trabalho é estudar o efeito causado pelo refluxo venoso das veias da panturrilha em pacientes portadores de varizes primárias, através da pletismografia a ar.

\section{Métodos}

Nos meses de abril e maio de 2004, foram avaliados 120 pacientes consecutivos, num total de 240 membros inferiores estudados. Após avaliação clínica, os pacientes foram submetidos ao mapeamento dúplex colorido, sendo divididos em dois grupos de 60 pacientes. $\mathrm{O}$ primeiro grupo apresentava refluxo das veias musculares da panturrilha (veias surais e geniculares), enquanto o segundo grupo não apresentava refluxo nessas veias. Utilizamos, como critério de refluxo, o fluxo venoso retrógrado que persiste por 0,5 segundo ou mais após a manobra de compressão ou de Valsalva ${ }^{9-11}$. Uma vez identificados os grupos, os pacientes, consecutivamente, foram avaliados quantitativamente através da pletismografia a ar. Utilizamos o protocolo de exame descrito por Nicolaides ${ }^{12}$ para a realização desse exame. Todos os pacientes eram sintomáticos e foram classificados entre C2 e C4 pela classificação clínica, etiológica, anatômica e fisiopatológica (CEAP) (Tabela 1) ${ }^{13}$. Não apresentavam outra doença concomitante nos sistemas venoso e arterial que pudesse interferir nos resultados. 


\section{Classificação CEAP}

Os pacientes que foram incluídos assinaram o termo de consentimento livre e esclarecido, segundo a Resolução 196/96 e a Resolução 251/97 do Conselho Nacional de Saúde, bem como a Declaração de Helsinki VI e o Código de Nuremberg. O estudo obteve aprovação do Comitê de Ética e Pesquisa da Universidade Estadual de Ciências da Saúde de Alagoas - Escola de Ciências Médicas de Alagoas (UNCISAL/ECMAL), Maceió, AL.

\section{Mapeamento dúplex}

Todos os pacientes foram examinados pelo mapeamento dúplex, e foram utilizadas sondas lineares de alta freqüência (7,5 ou $10 \mathrm{MHz}$ ) com modo B (imagem bidimensional). Utilizamos o Doppler pulsado (fluxo venoso) e o mapeamento a cores para o estudo das varizes tronculares primárias. $\mathrm{O}$ aparelho utilizado foi o HP Image Point Multispeciality Ultrasound System, da Hewlett Packard, 1999.

Inicialmente, cada paciente foi estudado em decúbito dorsal, com rotação externa e ligeira flexão da perna. O exame iniciou-se ao nível da prega inguinal, realizando-se varreduras transversais para o estudo da perviedade e para avaliação dos diâmetros venosos sob compressão segmentar ao longo de toda a extremidade. Em seguida, foi feita varredura longitudinal para estudo do padrão de onda e das características do fluxo, avaliando respostas aos movimentos respiratórios (inspiração e expiração), à compressão distal e à manobra de Valsalva.
O estudo das veias safena interna e externa foi feito com paciente em posição ortostática, sob compressão segmentar, utilizando-se transdutores de $10 \mathrm{MHz}$. Foram avaliadas as junções safeno-femoral e safenopoplítea e as tributárias da crossa, incluindo, nesse estudo, a avaliação das veias perfurantes.

Os segmentos infrageniculares (veias fibulares, tibiais anterior e posterior), bem com as veias da panturrilha (surais e geniculares), foram estudados em toda a sua extensão e submetidos a compressões segmentares, em cortes longitudinais e transversais, sendo avaliados segundo os principais tópicos: perviedade, compressibilidade, textura, diâmetro e características do fluxo.

\section{Pletismografia a ar}

Após avaliação pelo mapeamento dúplex, todos os pacientes foram submetidos a análise da função da bomba muscular da panturrilha por meio da pletismografia a ar com o aparelho SDV 3000 da marca Angiotec, com calibração automatizada por computador, fabricado no Brasil.

Para a realização do exame, a temperatura do laboratório permaneceu entre 22 a $24{ }^{\circ} \mathrm{C}$. A maca de exame foi colocada a uma altura de $55 \mathrm{~cm}$ para facilitar a mobilização da posição supina para a ortostática.

O pletismógrafo consiste em aparelho, conectado a um computador, e mangueira de poliuretano, conectada a manguito com $35 \mathrm{~cm}$ de comprimento e aproximadamente 5 litros de capacidade.

Tabela 1 - Número de pacientes dentro da classificação CEAP

\begin{tabular}{|c|c|c|c|c|c|c|c|}
\hline \multirow[b]{2}{*}{ Refluxo nas veias da panturrilha } & \multirow{2}{*}{$\begin{array}{c}\text { Pacientes } \\
\mathbf{n} \\
\end{array}$} & \multicolumn{2}{|c|}{$\mathrm{C} 2$} & \multicolumn{2}{|c|}{$\mathrm{C} 3$} & \multicolumn{2}{|c|}{$\mathrm{C} 4$} \\
\hline & & $\mathbf{n}$ & $\%$ & $\mathbf{n}$ & $\%$ & $\mathbf{n}$ & $\%$ \\
\hline Sim & 60 & 3 & 5 & 20 & 33,33 & 37 & 61,67 \\
\hline Não & 60 & 42 & 70 & 17 & 28,33 & 1 & 1,667 \\
\hline Total & 120 & 45 & 37,5 & 37 & 30,83 & 38 & 31,67 \\
\hline
\end{tabular}

$\mathrm{CEAP}=$ classificação clínica, etiológica, anatômica e fisiopatológica; $\mathrm{n}=$ número; $\%$ = percentual 
O exame é realizado com o paciente em posição supina, estando a perna elevada a $45^{\circ}$ e o joelho levemente fletido, para esvaziamento das veias. O pé é apoiado em suporte com altura de $20 \mathrm{~cm}$.

Coloca-se o manguito pneumático na perna do paciente desde o joelho até o tornozelo, não excedendo os limites da perna, permitindo, assim, bom contato do manguito com a pele. Este promove uma mínima oclusão das veias superficiais da perna.

Inicia-se, assim, a calibragem do aparelho de forma automática. Cuidados são tomados para que o manguito não fique em contato com o suporte de elevação do pé nem com a mesa de exame, a fim de não alterar a leitura pelo aparelho. Após comando eletrônico, o manguito é inflado automaticamente até pressão selecionada de $6 \mathrm{mmHg}$, e os dados são transmitidos ao transdutor de pressão, localizado no aparelho, amplificando o sinal e registrando-o em forma de gráfico na tela do computador.

Um valor basal de volume é obtido e, então, o paciente é solicitado a levantar-se auxiliado pelo examinador, apoiando-se, na posição de pé, no membro não avaliado.

Utiliza-se um andador no qual o paciente apóia as mãos. O gráfico mostra elevação da curva correspondendo ao aumento do volume de sangue venoso na perna. A curva do gráfico atinge nível máximo, formando um platô, indicando que as veias estão cheias. A diferença entre o volume inicial e o volume no platô representa o volume venoso (VV) funcional.

$\mathrm{Na}$ seqüência, o paciente é solicitado a apoiar-se com os dois pés no chão e a realizar um movimento de flexão plantar, provocando a contração da musculatura da panturrilha, retornando, a seguir, à posição inicial (repouso). A queda que se observa na curva do gráfico corresponde ao volume ejetado (VE), resultado da contração muscular da panturrilha.

Após esse movimento, um novo platô é atingido (geralmente mais elevado do que o inicial), e solicita-se que o paciente realize 10 movimentos de flexão plantar numa velocidade de um movimento por segundo. $\mathrm{O}$ decréscimo de volume observado é também anotado. $\mathrm{O}$ volume residual (VR) é calculado a partir do valor basal final em relação ao volume restante ao término dos movimentos.

Terminadas as flexões plantares, o paciente é solicitado a retornar à posição de repouso e relaxamento da perna examinada até atingir-se um novo platô de enchimento venoso. Findo o nivelamento, com auxílio do examinador, o paciente retorna à posição supina na mesa de exame com o pé sobre o suporte, e registra-se o esvaziamento total do membro, mostrado por um nivelamento do gráfico correspondendo ao volume final (VF) (Figura 1).

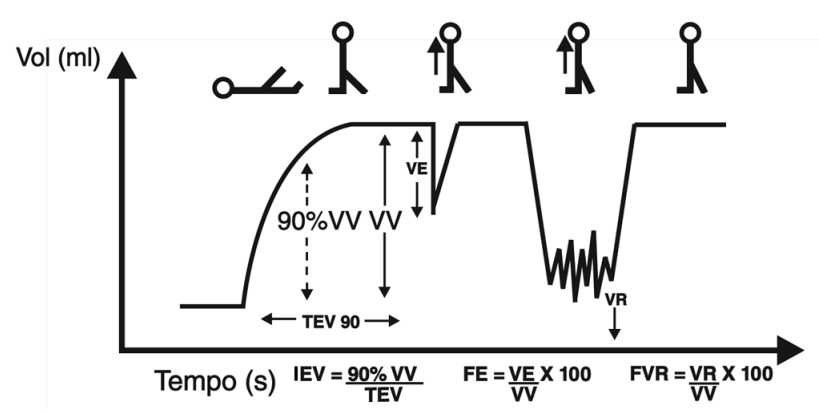

$\mathrm{FE}=$ fração de ejeção; FVR = fração do volume residual; IEV = índice de enchimento venoso; TEV = tempo de enchimento venoso; $\mathrm{VE}=$ volume de ejeção; $\mathrm{VR}=$ volume residual; $\mathrm{VV}=$ volume venoso.

Figura 1 - Curva pletismográfica com exercícios correspondentes

O gráfico volume/tempo é visualizado na tela do computador. Uma vez traçado o gráfico, são feitas marcações com o cursor do programa de computador correspondentes ao volume zero inicial, ao $\mathrm{VV}$, ao $\mathrm{VE}$ mínimo, ao volume de reenchimento (VRe), ao volume residual mínimo (VRmin) e, por fim, ao volume final. O programa marca automaticamente o tempo gasto para alcançar 90\% do volume venoso $(90 \% \mathrm{VV})$.

Realizadas as marcações no gráfico, o computador processa os cálculos automaticamente para: índice de enchimento venoso (IEV), fração de ejeção (FE) e fração do volume residual (FVR), sendo esses valores mostrados na tela do computador.

O IEV é a variação de volume produzida no membro dividida pelo tempo correspondente, em segundos, na 
passagem da posição deitada para a posição de pé. É calculado através da fórmula: IEV $=90 \% \mathrm{x}$ VV/TEV90, expresso em $\mathrm{mL} / \mathrm{s}$.

Estudamos a função de bomba da panturrilha através dos dados obtidos pelas variáveis FE e FVR.

A FE nos fornece a idéia do retorno venoso durante a atividade física e das alterações que resultam em menos sangue ejetado. A FE é a porcentagem do volume venoso total ejetado em uma única contração da musculatura da panturrilha, sendo calculada pela fórmula: $\mathrm{FE}=(\mathrm{VE} / \mathrm{VV}) \times 100$.

A FVR correlaciona-se linearmente com a pressão venosa ambulatorial, sendo, porém, medida de forma não-invasiva através da fórmula: $F V R=(V R / V V) x$ $100^{14}$.

\section{Análise estatística}

$\mathrm{Na}$ análise estatística, foram utilizados o teste de hipótese (teste qui-quadrado), razão de chances (odds ratio) e intervalos de confiança para as variáveis $\mathrm{FE}$ (direita e esquerda) e FVR (direita e esquerda) e VE (direito e esquerdo). Para a análise descritiva, foram utilizadas tabelas com freqüências absolutas e percentuais para as variáveis estudadas. Utilizou-se também uma técnica de análise multivariada para freqüências, a análise de correspondência, para determinar o diagrama de influência entre as freqüências das variáveis refluxo, FE (direita e esquerda) e FVR (direita e esquerda) e VE (direito e esquerdo).

O erro tipo I (nível de significância $=\alpha$ ) foi admitido em $5 \%$. Portanto, se $\mathrm{p} \leq 0,05$, rejeita-se a hipótese nula e considera-se o resultado significativo.

Os cálculos foram realizados com o auxílio do programa de computador SPSS 8.0 for Windows, Chicago, 1995.

\section{Resultados}

Dos pacientes estudados, 10,8\% (13/120) eram do sexo masculino e $89,2 \%$ (107/102) do sexo feminino. A idade média foi de 35 anos ( \pm 3 anos). No grupo dos pacientes com refluxo das veias surais e geniculares, foram classificados três $(5 \%)$ pacientes na categoria $\mathrm{C} 2$, $20(33,3 \%)$ na $\mathrm{C} 3$ e $37(61,6 \%)$ na C4. No grupo sem refluxo, foram encontrados $42(70 \%)$ pacientes na categoria $\mathrm{C} 2,17(28,3 \%)$ na $\mathrm{C} 3$ e um $(1,6 \%)$ na $\mathrm{C} 4$ (Tabela 1).

Em nossa amostra, verificamos que o teste quiquadrado revelou associação entre refluxo venoso das veias musculares da panturrilha e FE $>60 \%$, independentemente do lado estudado.

Entre os pacientes que apresentaram FE $>60 \%$ no membro inferior esquerdo, $15,5 \%$ (9/58) tiveram refluxo nas veias musculares da panturrilha esquerda, avaliadas pelo estudo de eco-Doppler, enquanto 84,5\% (49/58) não apresentaram este refluxo. Por outro lado, entre os que apresentaram $\mathrm{FE}<60 \%$ na panturrilha esquerda, a maioria $(82,3 \%$, isto é, $51 / 66$ tinha refluxo, em contraste com $17,7 \%$ (11/66), que não apresentaram refluxo, conforme mostra a Tabela 2.

Quando analisamos o lado esquerdo, o teste quiquadrado revelou associação significativa entre refluxo e FE (Tabela 2), sendo provável a presença de refluxo no grupo com FE esquerda abaixo de $60 \%$ (com $82,3 \%$ ). A razão de chances mostrou que um indivíduo com FE esquerda acima de $60 \%$ tem $25(1 / 0,04)$ vezes menos chances de ter refluxo das veias da panturrilha. Tal dado baseia-se no fato de que esse risco está em um intervalo menor do que 1 , isto é, compreendido entre 0,015 e 0,104 , com um intervalo de confiança de $95 \%$.

O mesmo ocorreu com o membro inferior direito em relação à $\mathrm{FE}$. Entre os pacientes que tiveram $\mathrm{FE}>60 \%$, a minoria $(28,8 \%$, isto é, $10 / 53)$ apresentou refluxo das veias da panturrilha, enquanto a maioria $(71,2 \%$, isto é, 43/53) não apresentou refluxo. Já nos casos de pacientes com $\mathrm{FE}<60 \%$, a maioria $(74,6 \%$, isto é, $50 / 67)$ teve refluxo, e os demais $25,4 \%$ (17/67) não tiveram refluxo (Tabela 2).

$\mathrm{O}$ teste qui-quadrado revelou associação significativa entre refluxo e FE da perna direita. O refluxo foi mais presente no grupo com $\mathrm{FE}$ da perna direita abaixo de $60 \%$. A razão de chances mostrou que indivíduos com FE da perna direita abaixo de $60 \%$ têm 12,5 $(1 / 0,08)$ vezes mais chances de apresentar refluxo, podendo este risco estar compreendido entre 0,033 a 0,191 com $95 \%$ de intervalo de confiança. 
Na avaliação da FVR da perna esquerda, encontra$\operatorname{mos} 28,8 \%(15 / 52)$ de ocorrência de refluxo nas veias da panturrilha com valores abaixo de $35 \%$, e $62,5 \%$ (10/16) de refluxo com valores acima de $60 \%$. No grupo sem refluxo, tivemos $71,2 \%$ (37/52) abaixo de $35 \%$, e $37,5 \%$ (6/16) acima de 60\% (Tabela 2).

O valor qui-quadrado 5,96 (com valor de $\mathrm{p}=0,015$ ) informou que há associação entre FVR da perna esquerda e o refluxo, ou seja, que são fatores que se influenciam. A razão de chances informou que há aproximadamente 4 vezes (exatas 4,11) mais chances de ocorrer refluxo quando a FVR esquerda está acima de $60 \%$. Este resultado é significativo.

O mesmo foi observado para o membro inferior direito. Neste grupo, onde se detectou FVR $<35 \%$, $29,2 \%$ (14/48) tiveram refluxo e $70,8 \%$ (34/48) não tiveram refluxo das veias soleares e gastrocnêmias. Entretanto, para valores de FVR acima de 60\%, 86,7\% $(13 / 15)$ tiveram refluxo, em contraste com 13,3\% (2/15) que não tiveram refluxo (Tabela 2).

O valor qui-quadrado 1,68 (com valor de $p=0,140$ ) informou que a FVR direita e o refluxo são dependentes, ou seja, que estão associados. A razão de chances informou que há cerca de 2 vezes (exatas 1,75) mais chances de não ocorrer refluxo quando a FVR direita é baixa (está abaixo de 35\%). Este resultado não é significativo.

Foi avaliado o IEV nos grupos estudados. Em perna esquerda, índices acima de $2 \mathrm{~mL} / \mathrm{s}$ ocorreram em membro com refluxo em 39,3\% (11/28) e em membro sem refluxo em 60,7\% (17/28) dos casos. Entre os índices abaixo de $2 \mathrm{~mL} / \mathrm{s}, 53,3 \%$ (49/92) apresentaram refluxo e $46,7 \%$ (43/92) não apresentaram refluxo das veias da panturrilha (Tabela 3).

De acordo com os dados apresentados, não houve associação significativa entre o IEV da perna esquerda e a ocorrência de refluxo. O teste qui-quadrado mostrou que a ocorrência do refluxo apresentou proporção igual nos grupos acima e abaixo de $2 \mathrm{~mL} / \mathrm{s}$. A razão de chances de 0,57 mostrou que a ocorrência do refluxo foi igual nos indivíduos com IEV alto ou baixo.

$\mathrm{Na}$ mesma análise, porém para a perna direita, $41,9 \%$ (13/31) tiveram refluxo e $58,1 \%$ (18/31) não tiveram refluxo nas veias da panturrilha em índices acima de $2 \mathrm{~mL} / \mathrm{s}$. Da mesma forma, para índices abaixo de $2 \mathrm{~mL} / \mathrm{s}, 52,8 \%$ (47/89) apresentaram refluxo e 47,2\% (42/89) não apresentaram refluxo (Tabela 3). Não houve, portanto, associação significativa entre o IEV da

Tabela 2 - Avaliação da fração de ejeção e da fração do volume residual para pernas direita e esquerda

\begin{tabular}{|c|c|c|c|c|c|c|c|c|c|c|c|c|c|c|c|c|}
\hline \multirow{4}{*}{$\begin{array}{l}\text { Refluxo nas veias } \\
\text { da panturrilha }\end{array}$} & \multicolumn{8}{|c|}{ Fração de ejeção (FE) } & \multicolumn{8}{|c|}{ Fração do volume residual (FVR) } \\
\hline & \multicolumn{4}{|c|}{$>60 \%$} & \multicolumn{4}{|c|}{$<60 \%$} & \multicolumn{4}{|c|}{$<35 \%$} & \multicolumn{4}{|c|}{$>60 \%$} \\
\hline & \multicolumn{2}{|c|}{ Dir } & \multicolumn{2}{|c|}{ Esq } & \multicolumn{2}{|c|}{ Dir } & \multicolumn{2}{|c|}{ Esq } & \multicolumn{2}{|c|}{ Dir } & \multicolumn{2}{|c|}{ Esq } & \multicolumn{2}{|c|}{ Dir } & \multicolumn{2}{|c|}{ Esq } \\
\hline & $\mathbf{n}$ & $\%$ & $\mathbf{n}$ & $\%$ & $\mathbf{n}$ & $\%$ & $\mathbf{n}$ & $\%$ & $\mathbf{n}$ & $\%$ & $\mathbf{n}$ & $\%$ & $\mathbf{n}$ & $\%$ & $\mathbf{n}$ & $\%$ \\
\hline Sim & 10 & 28,8 & 9 & 15,5 & 50 & 74,6 & 55 & 82,3 & 14 & 29,2 & 15 & 28,8 & 13 & 86,7 & 10 & 62,5 \\
\hline Não & 43 & 71,2 & 49 & 84,5 & 17 & 25,4 & 11 & 17,7 & 34 & 70,8 & 37 & 71,2 & 2 & 13,3 & 6 & 37,5 \\
\hline Total & 53 & 100 & 58 & 100 & 67 & 100 & 66 & 100 & 48 & 100 & 52 & 100 & 15 & 100 & 16 & 100 \\
\hline
\end{tabular}

Perna direita (FE) - razão de chances $=0,08($ IC95\% 0,033 a 0,191); qui-quadrado $=36,8 ; \mathrm{p}<0,001$;

Perna esquerda (FE) - razão de chances $=0,04$ (IC95\% 0,015 a 0,104); qui-quadrado $=26,77 ; \mathrm{p}<0,001$;

FE - Normal $=<60 \%$; Insuficiente $=>60 \%$;

Perna direita (FVR) - razão de chances = 0,57 (IC95\% 0,24 a 1,34); qui-quadrado = 1,68; $\mathrm{p}=0,140$;

Perna esquerda (FVR) - razão de chances $=4,11($ IC95\% 1,27 a 13,33); qui-quadrado $=5,96 ; \mathrm{p}=0,015$;

FVR - Normal $=<35 \%$; Alta $=>60 \%$ 
perna direita e a ocorrência do refluxo. O teste quiquadrado mostrou que a ocorrência do refluxo apresenta proporção igual nos grupos acima e abaixo de 2 $\mathrm{mL} / \mathrm{s}$. A razão de chances de 0,57 mostrou que a ocorrência do refluxo foi estatisticamente igual nos grupos com IEV acima e abaixo de $2 \mathrm{~mL} / \mathrm{s}$.

Outra variável estudada foi o VE. Para a perna esquerda, 25,6\% (11/43) das ocorrências com VE entre 50 a $150 \mathrm{~mL}$ apresentaram refluxo nas veias da panturrilha, e 74,4\% (32/43) não apresentaram refluxo. Por outro lado, entre os pacientes que apresentaram VE acima de $150 \mathrm{~mL}$,

$63,6 \%$ (49/77) tiveram refluxo e 36,4\% (28/77) não tiveram refluxo (Tabela 3). Deste modo, o teste quiquadrado mostrou que os dados observados apresentaram evidências estatísticas de que houve associação entre o VE na perna esquerda e o refluxo. A razão de chances revelou que há 5 vezes mais chances de ocorrer refluxo com VE entre 50 e $150 \mathrm{~mL}$.

Na perna direita, observou-se que 29,5\% (13/44) dos pacientes com VE entre 50 e $150 \mathrm{~mL}$ apresentaram refluxo nas veias da panturrilha, enquanto 70,5\% (31/ 44) não apresentaram refluxo. Já no grupo com VE acima de $150 \mathrm{~mL}, 61,8 \%$ (47/76) tiveram refluxo, em contraste com 38,2\% (29/76) sem refluxo das veias surais e geniculares (Tabela 3). Na Tabela 3, o teste qui-quadrado mostrou que os dados observados não apresentaram evidência estatística de associação entre VE e refluxo. A razão de chances mostrou que há 3,9 vezes mais chances de ocorrer refluxo com VE de 50 a $150 \mathrm{~mL}$, e esta razão de chances mostrou que o seu intervalo de $95 \%$ de confiança foi superior ao risco unitário (o intervalo está acima de 1).

A Figura 2 refere-se ao resultado da técnica multivariada para análise de correspondência aplicada aos dados agrupados na Tabela 2, referentes aos 120 pacientes estudados.

Os fatores que mais estão próximos ao refluxo são a FE abaixo de $60 \%$ e a FVR acima de $35 \%$.

Os pacientes com FE abaixo de $60 \%$ estão mais propensos a terem refluxo nas veias da panturrilha, enquanto o grupo de pacientes com VE nas pernas esquerda e direita acima do volume normal está mais propenso a ter dilatação das veias da panturrilha.

\section{Discussão}

A pletismografia a $\operatorname{ar}^{7}$ e o mapeamento dúplex ${ }^{8,15}$ são, atualmente, as ferramentas com a maior possibilidade de medir a variação volumétrica absoluta e a

Tabela 3 - Avaliação do índice de enchimento venoso e do volume ejetado para pernas direita e esquerda

\begin{tabular}{|c|c|c|c|c|c|c|c|c|c|c|c|c|c|c|c|c|}
\hline \multirow{4}{*}{$\begin{array}{l}\text { Refluxo nas veias } \\
\text { dapanturrilha }\end{array}$} & \multicolumn{8}{|c|}{ Índice de enchimento venoso (IEV) } & \multicolumn{8}{|c|}{ Volume ejetado (VE) } \\
\hline & \multicolumn{4}{|c|}{$>2 \mathrm{~mL} / \mathrm{s}$} & \multicolumn{4}{|c|}{$<2 \mathrm{~mL} / \mathrm{s}$} & \multicolumn{4}{|c|}{ Normal } & \multicolumn{4}{|c|}{ Acima } \\
\hline & \multicolumn{2}{|c|}{ Dir } & \multicolumn{2}{|c|}{ Esq } & \multicolumn{2}{|c|}{ Dir } & \multicolumn{2}{|c|}{ Esq } & \multicolumn{2}{|c|}{ Dir } & \multicolumn{2}{|c|}{ Esq } & \multicolumn{2}{|c|}{ Dir } & \multicolumn{2}{|c|}{ Esq } \\
\hline & $\mathbf{n}$ & $\%$ & n & $\%$ & n & $\%$ & $\mathbf{n}$ & $\%$ & $\mathbf{n}$ & $\%$ & n & $\%$ & $\mathbf{n}$ & $\%$ & $\mathbf{n}$ & $\%$ \\
\hline $\operatorname{Sim}$ & 13 & 41,9 & 11 & 39,3 & 47 & 52,8 & 49 & 53,3 & 13 & 29,5 & 11 & 25,6 & 47 & 61,8 & 49 & 63,6 \\
\hline Não & 18 & 58,1 & 17 & 60,7 & 42 & 47,2 & 43 & 46,7 & 31 & 70,5 & 32 & 74,4 & 29 & 38,2 & 28 & 36,4 \\
\hline Total & 31 & 100 & 28 & 100 & 89 & 100 & 92 & 100 & 44 & 100 & 43 & 100 & 76 & 100 & 77 & 100 \\
\hline
\end{tabular}

Perna direita (IEV) - razão de chances $=0,57($ IC95\% 0,24 a 1,34); qui-quadrado $=1,68 ; \mathrm{p}=0,140$;

Perna esquerda (IEV) - razão de chances $=0,57(\mathrm{IC} 95 \% 0,24$ a 1,34); qui-quadrado $=1,68 ; \mathrm{p}=0,140$;

IEV - sem refluxo $=<2 \mathrm{~mL} / \mathrm{s} ;$ com refluxo $=>2 \mathrm{~mL} / \mathrm{s}$

Perna direita (VE) - razão de chances $=3,9$ (IC95\% 1,74 a 8,54); qui-quadrado = 11,63; $\mathrm{p}=0,001$;

Perna esquerda (VE) - razão de chances $=5,0($ IC95\% 2,20 a 11,11); qui-quadrado $=15,98 ; \mathrm{p}<0,001$;

$\mathrm{VE}-$ Normal $=50-150 \mathrm{~mL} ;$ Acima $=>150 \mathrm{~mL}$ 


\section{Quantificação de Categoria}

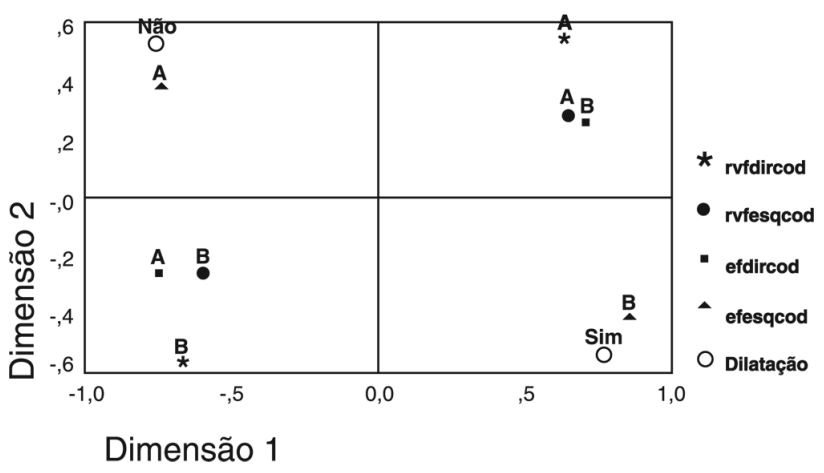

Figura 2 - Análise de correspondência para fração de ejeção da perna direita (efdircod), fração de ejeção da perna esquerda (efesqcod), fração do volume residual da perna direita (rvfdircod), fração do volume residual da perna esquerda (rvfesqcod) nos grupos com refluxo e sem refluxo

visualização das veias in situ de forma não-invasiva. São métodos simples, não-invasivos e reprodutíveis na avaliação da hemodinâmica venosa dos membros inferiores $^{3,5}$. Os dados avaliados por meio da pletismografia a ar são quantitativos tanto para o refluxo valvular como para a função de bomba muscular da panturrilha, enquanto o mapeamento dúplex ${ }^{16}$ avalia os mesmos dados, porém mostra detalhes anatômicos, de forma qualitativa e em segmentos com refluxo e obstrução ${ }^{17}$.

Ao contrário do mapeamento dúplex, que é um exame dependente do examinador, a pletismografia a ar pode ser realizada por pessoas qualificadas e treinadas no uso do equipamento e é um exame mais dependente do paciente do que do examinador, pois qualquer variação de postura (como, por exemplo, uma contratura muscular mais acentuada ou uma flexão de pés feita de forma parcial) alterará a forma do gráfico. Uma vez alterado o gráfico, os valores finais absolutos ficarão comprometidos e, por conseguinte, será feita marcação do gráfico de forma errada, resultando em interpretação equivocada, não condizente com a realidade fisiopatológica do indivíduo examinado. Por essa razão, todo paciente que apresentar alguma deformidade ou dificuldade para flexionar os pés terá seu exame comprometido, além de não conseguir completá-lo. Alguns pacientes têm dificuldade inclusive em compre- ender os exercícios propostos durante o exame. Muitas vezes, ao descer da mesa de exame, alguns pacientes acabam por apoiar-se nos dois pés, promovendo esvaziamento venoso parcial da panturrilha.

Para alguns pacientes, o exame torna-se cansativo. Nos casos em que o enchimento venoso é mais lento, o paciente deve permanecer em pé sobre uma única perna, apoiando-se apenas com as mãos, por tempo mais prolongado, podendo apresentar dores musculares e não esperar o término do exame. Além disso, pacientes mais idosos e portadores de doenças de origem reumática, osteomuscular ou cardíaca têm maior dificuldade na realização do exame.

Uma vez que a mesa de exame tem altura padronizada de cerca de $55 \mathrm{~cm}$, pacientes com alturas acima de $1,80 \mathrm{~m}$ ou abaixo de $1,50 \mathrm{~m}$ apresentam alguma dificuldade para levantarem-se da mesa de exame sem alterar, de forma comprometedora, a curva pletismográfica.

Há uma reprodutibilidade das medidas expressas em percentuais para IEV, FE e FVR, todas tendo um coeficiente de variação menor do que $10 \%{ }^{18}$. Nossos resultados demonstram não haver relação significativa entre o IEV e o refluxo nas veias da panturrilha, já que, em perna esquerda, $60,7 \%$ dos pacientes que apresentaram IEV acima de $2 \mathrm{~mL} / \mathrm{s}$ não tiveram refluxo. Nicolaides $^{12}$ encontrou IEV acima de $2 \mathrm{~mL} / \mathrm{s}$ associado à diminuição da FE com valores abaixo de $60 \%{ }^{19}$, apresentando sinais significativos de refluxo venoso, tendo inclusive, em tais pacientes, um potencial para desenvolvimento de ulcerações em membros inferiores ${ }^{20}$.

Os resultados obtidos por esses autores demonstram o desenvolvimento da insuficiência venosa ${ }^{8}$ pela alteração dos valores absolutos nos pacientes sintomáticos, porém sem haver manifestação clínica evidente ${ }^{12}$.

A hipertensão venosa manifesta-se através de sinais e sintomas que já foram bem estabelecidos por outros autores ${ }^{21,22}$. O ponto em que parece haver discordância é que, no nosso trabalho, mesmo não havendo sinais externos que possam levantar suspeita de hipertensão venosa, a FVR (que, para alguns autores ${ }^{16,21}$, se relaciona com a pressão venosa ambulatorial) ocorreu em $86,7 \%$ dos pacientes que apresentaram níveis acima de 
$60 \%$, sendo este dado estatisticamente relevante. Há, porém, concordância com a literatura quanto ao fato de que o VE não apresenta diferenças estatisticamente significativas para ambos os sexos ${ }^{23}$. Embora a complacência venosa esteja alterada, em função do refluxo encontrado, não há influência para a hemodinâmica venosa $^{24}$.

A pletismografia a ar permite uma análise completa da hemodinâmica venosa de todo o membro ${ }^{25}$, que pode ser somada aos dados obtidos com o mapeamento dúplex, que permite analisar anormalidades em veias individualizadas ${ }^{8}$.

$\mathrm{Na}$ insuficiência venosa em estágio inicial, há casos em que os sinais clínicos não apresentam correspondência direta com a sintomatologia referida pelos pacientes $^{22}$. Para esses casos, a pletismografia a $\mathrm{ar}^{26}$ tem importante papel na identificação, quantificação e diferenciação dos estágios iniciais da insuficiência venosa. Um exame bem indicado oferece informações valiosas que poderão orientar o tratamento, bem como seu acompanhamento.

As variações encontradas ao longo do tratamento identificarão quantitativamente os pontos alvos a serem atingidos e o que deverá ser modificado em termos da abordagem terapêutica, proporcionando um prognóstico mais aproximado do real e oferecendo mais conforto e menos ansiedade ao médico e ao seu paciente. A pletismografia a ar é um exame cuja aplicabilidade técnica torna-se simples, sendo um exame não-invasivo de baixo custo que apresenta resultados de importância na evolução e no acompanhamento dos pacientes portadores de doenças venosas.

Sendo assim, conclui-se, com este trabalho, que, havendo refluxo nas veias da panturrilha como resultado primário da insuficiência venosa, será afetada a hemodinâmica do retorno venoso realizado através da contração da bomba muscular da panturrilha. Não havendo outros comemorativos clínicos para justificar a sintomatologia apresentada e tendo sido afastadas doenças de natureza diversa, encontramos respostas na insuficiência da bomba muscular da panturrilha causada inicialmente pela alteração estrutural de suas veias.
Entretanto, não devemos parar por aqui. Apesar de os dados apresentados terem a sua significância, há muito o que pesquisar, há muito o que descobrir.

\section{Referências}

1. Ruckley CV, Evans CJ, Allan PL, Lee AJ, Fowkes FG. Chronic venous insufficiency: clinical and duplex correlations. The Edinburgh Vein Study of venous disorders in the general population. J Vasc Surg. 2002;36:520-5.

2. Zsoter T. Cronin RF. Venous distensibility in patients with varicose veins. Can Med Assoc J. 1966;94:1293-7.

3. Bollinger A, Leu AJ, Hoffmann U, Franzeck UK. Microvascular changes in venous disease: an update. Angiology. 1997;48:27-32.

4. Morano JU, Raju S. Chronic venous insufficiency : assessment with descending venography. Radiology. 1990;174:441-4.

5. Shami SK, Sarin S, Cheatle TR, Scurr JH, Smith PD. Venous ulcers and the superficial venous system. J Vasc Surg. 1993; 17:487-90.

6. Becker F. Mechanisms, epidemiology and clinical evaluation of venous insufficiency of the lower limbs. Rev Prat. 1994;44:726-31.

7. Christopoulos DG, Nicolaides AN, Szendro G, Irvine AT, Bull ML, Eastcott HH. Air-plethysmography and the effect of elastic compression on venous hemodynamics of the leg. $\mathbf{J}$ Vasc Surg. 1987;5:148-59.

8. Bradbury AW, Stonebridge PA, Ruckley CV, Beggs I. Recurrent varicose veins: correlation between preoperative clinical and hand-held Doppler ultrasonographic examination, and anatomical findings at surgery. Br J Surg. 1993;80:849-51.

9. Kalodiki E, Calahoras L, Nicholaides AN. Make it easy: duplex examination of the venous system. Phlebology. 1993;8:17-21.

10. Jiang P, van Rij AM, Christie R, Hill G, Solomon C, Thomson I. Recurrent varicose veins: patterns of reflux and clinical severity. Cardiovasc Surg. 1999;7:332-9.

11. Jiang P, van Rij AM, Christie RA, Hill GB, Thomson IA. Venous physiology in the different patterns of recurrent varicose veins and the relationship to clinical severity. Cardiovasc Surg. 2000;8:130-6.

12. Nicolaides AN. Diagnostic evaluation of pacients with cronic venous insufficiency. In: Rutherford RB, ed. Vascular surgery. 3th ed. Philadelphia: WB Saunders; 1989. p. 1583-601.

13. Porter J, Moneta GL. Reporting standards in venous disease: an update. International Consensus Committee on Chronic Venous Disease. J Vasc Surg. 1995;21:635-45.

14. Rutherford RB. Vascular surgery. 4th ed. Philadelphia Saunders; 1995. p. 1772.

15. Christopoulos DG, Nicolaides AN, Szendro G, Irvine AT, Bull ML, Eastcott HH. Air-plethysmography and the effect of elastic compression on venous hemodynamics of the leg. J Vasc Surg. 1987;5:148-59. 
16. van Rij AM, Jiang P, Solomon C, Christie RA, Hill GB. Recurrence after varicose vein surgery: A prospective long-term clinical study with duplex ultrasound scanning and air plethysmography. J Vasc Surg. 2003;38:935-43.

17. Harada RN, Katz ML, Comerota A. A noninvasive screening test to detect "critical" deep venous reflux. J Vasc Surg. 1995;22:532-7.

18. Christopoulos D, Nicolaides NA, Air plethysmography. In: Raju S, Villavicencio JL. Surgical management of venous disease. Baltimore: Willians \& Wilkins; 1997. p. 93.

19. Belcaro G, Nicolaides AN, Veller M. Assessment of the venous and lymphatic systems. In: Belcaro G, Nicolaides AN, eds. Venous disorders: a manual of diagnosis and treatment. Baltimore: Saunders; 1995. p. 41.

20. Comerota AJ, Harada RN, Eze AR, Katz ML. Air plethysmography: a clinical review. Int Angiol. 1995;14:45-52.

21. Christopoulos D, Nicolaides AN, Szendro G. Venous reflux: quantification and correlation with the clinical severity of chronic venous disease. Br J Surg. 1988;75:352-6.

22. Bradbury A, Evans C, Allan P, Lee A, Ruckley CV, Fowkes FG. What are the symptoms of varicose veins? Edinburgh Vein Study cross sectional population survey. BMJ. 1999;318: 353-6.
23. Evans CJ, Fowkes FG, Ruckley CV, Lee AJ. Prevalence of varicose veins and chronic venous insufficiency in men and women in the general population: Edinburgh Vein Study. J Epidemiol Community Health. 1999;53:149-53.

24. Evans CJ, Allan PL, Lee AJ, Bradbury AW, Ruckley CV, Fowkes FG. Prevalence of venous reflux in the general population on duplex scanning: the Edinburgh vein study. $\mathbf{J}$ Vasc Surg. 1998;28:767-76.

25. Nicolaides AN, Summer DS, eds. Investigation of patients with deep vein thrombosis and chronic venous insufficiency. Los Angeles: Méd-orion; 1991. p. 9-61.

26. Christopoulos D, Nicolaides AN, Cook A, Irvine A, Galloway JM, Wilkinson A. Pathogenesis of venous ulceration in relation to the calf muscle pump function. Surgery. 1989;106:829-35.

Correspondência:

André de Araujo Sacchi

Rua Cônego Pequeno 615, Bairro Prata

CEP 58101-343 - Campina Grande, PB

Tel.: (83) 3321.2442, (83) 3321.1976, (38) 9913.0509

E-mail: aasacchi@uol.com.br 